\title{
Implementation of Incentive Mechanism in Participatory Sensing-Based River Management System
}

\author{
Tomomichi Hayakawa *, Teruhisa Hochin ${ }^{*}$, Tokuro Matsuo ${ }^{\dagger}$
}

\begin{abstract}
The illegal dumping of garbage, flooding, and embankment and bridge failures are some of the common problems related to rivers. To be able to tackle these issues effectively, it is necessary to understand and respond to them with speed. In addition, future measures are required. However, since river basins can be very large, it is difficult for the river management staff to notice and respond to all river-related issues promptly. In order to improve the system used in Japan for monitoring the areas around rivers, we collaborated with the Ministry of Land, Infrastructure and Transport Chubu Regional Development Bureau Shonai River Office. In particular, to enable river administrators to quickly understand the issues occurring around rivers and to help them resolve these issues quickly, we developed a River Information Management System (RIMS) that promotes information sharing by the local residents through smartphones. This RIMS consists of a River Information Application (RIA) and a Post Management System (PMS). The RIA is an application for posting river-related problems using a smartphone. On the other hand, the PMS can be used by the river administrators to view the problems posted and respond to them. The developed system was tested successfully by the river administrators and patrol staff of the river office in the Shonai River basin.
\end{abstract}

Keywords: River management, Participatory sensing, Gamification, Incentive Mechanism, Smartphone application

\section{Introduction}

In the areas surrounding river basins, the illegal dumping of garbage, flooding, and embankment and bridge failures are common problems. It is necessary to understand and respond to these problems quickly. However, since river basins can be very large, it is not possible for the river management staff to monitor and respond to all the issues rapidly. In order to streamline the monitoring system used by these personnel, it is essential to monitor a wide range of river basins

\footnotetext{
* Kyoto Institute of Technology, Kyoto, Japan

$\dagger$ Advanced Institute for Industrial Technology, Tokyo, Japan
} 
based on the information provided by the residents living around the rivers. However, when receiving such information from the residents living around the rivers, it is often the case that one cannot quickly understand the problem and ascertain its location based on orally provided information. The purpose of this study was to develop a River Information Management System (RIMS) that will allow river administrators to quickly grasp the problems occurring around rivers and promptly respond to them based on the information provided by the residents and members of cooperating organizations located near the rivers using smartphones. The primary problems and the approaches to them of the study are described as follow:

It is required to develop a system that can receive information accurately from residents around the river and then allows this information be used effectively by river administrators.

To this end, we developed the RIMS for accurately receiving information from the residents living around rivers by utilizing the GPS functionality of smartphones as well as their camera for posting river-related information.

The absence of messages does not necessarily mean that there are no problems. For instance, it can be that no one has noticed the problems that exist. The RIMS implements a check-in function in the form of a game. The river administrator can distinguish between places where nobody leans owing to the presence or absence of check-ins; this is true even in places for which there have been no posts.

In order to encourage the residents around rivers to use the RIMS for effectively obtaining information related to rivers, it is necessary to develop a method for attracting them. The RIMS grants points and show the point ranking.

The system described above was developed and tested for the Shonai River, which flows through Aichi and Gifu prefectures by collaborating with the Ministry of Land, Infrastructure and Transport Chubu Regional Development Bureau Shonai River Office.

The structure of the rest of the paper is as follows. Section 2 reviews previous studies on participatory sensing, gamification, and incentive mechanisms and describes how the developed RIMS is different from those reported previously. In Section 3, we describe the implementation of the RIMS for solving river-related problems. Section 4 describes the configuration of the RIMS while Section 5 describes the results of field tests of the RIMS performed at the Shonai River Office. Finally, Section 6 summarizes the results obtained.

\section{Related Work}

\subsection{Participatory Sensing}

Participatory sensing [1][2][3] is a method of collecting environmental data. In recent years, the widespread use of smartphones equipped with GPS receivers, temperature sensors, and cameras and the spread of low-cost wireless networks has made it possible for citizens to readily share sensed data. This phenomenon can be considered crowdsourcing, wherein information obtained from sensor devices by a plurality of users is sent to a common server. There have been several studies on behavior estimation [4][5] and position estimation [6] based on similarly collected sensor information. In addition, there has also been research on participants' sensing tasks [7]. 
FixMyStreet ${ }^{1}$ and SeeClickFix ${ }^{2}$ are existing participatory sensing services, which allow the user to report local problems (such as road damage and illegal dumping) to the administrative agencies through smartphones and the like. Participants can report problems such as road breakages, graffiti, street lamp failures, illegal dumping, noise, and barrier-free using text and photographs via dedicated applications installed on smartphones. The administration can then respond promptly to these problems in a timely manner. FixMyStreet is available in various municipalities in Handa City, Beppu City, Koriyama City, and Ikoma City in Japan.

The RIMS developed in this study is a participatory sensing service that is based on the use of smartphones. In existing participatory sensing services, the local government uses general-purpose off-the-shelf services. On the other hand, in case of the developed system, the services were developed based on the river administrators' requests. In particular, the local administrations in Japan are cautious about malicious postings that can be considered slanderous and mischievous, and there is a need for functions that allow for such postings to be dealt with.

\subsection{Gamification}

Gamification [8][9][10] is a method of motivating participants to take action by incorporating elements of games into activities that are not games. These elements can be a point system, ranking visualizations, badges, missions, and levels. The participants get deeper satisfaction by using services in which gamification has been incorporated.

The effectiveness of gamification has been confirmed through several studies. For instance, Ahn et al. [11] successfully used gamification to encourage users to join a crowdsourcing service for accurately labeling image data that already exists online.

The proposed RIMS implements a check-in function based on gamification to encourage its users to participate in data reporting. Many gamification-based crowdsourcing services exist already. However, there are no sensing services that exploit gamification.

\subsection{Incentive Mechanism}

Incentives are necessary for ensuring the active participation of the users. Providing monetary compensation to the users is one way of providing an incentive for participating. However, in order to ensure that the users participate in high-volume sensing, stronger motivation is necessary. This would lead to very high remunerations. We have studied ways of recruiting large numbers of users and increasing their motivation [12][13][14]. In addition, there have also been studies on how to improve the information gains of the sensing platform being used while focusing on reducing the overall sensing cost [15]. Methods that are not based on monetary compensation but on an incentive mechanism that incorporates gamification have also been reported [16]. The introduction of a gaming aspect such as levels, rankings, or a badge system can allow one to do away with monetary compensation.

The RIMS implements a point function as well as a point ranking function, both of which are incentive mechanisms based on gamification to ensure that the users participate more actively. The existing participatory sensing services do not incorporate such mechanisms.

\footnotetext{
1 FixMyStreet, https://www.fixmystreet.com/

2 SeeClickFix, http://seeclickfix.com/
} 


\section{Functions of Proposed System}

\subsection{Entry Function}

The RIMS consists of RIA and PMS. The RIA implements a posting function, which allows the users to inform the river administrators of any problems that have occurred in the vicinity of the river. The user who discovers a problem posts their location information (latitude and longitude) along with explanatory texts and photographs of the problem site to the PMS using the RIA. Based on these posts, the river administrator is able to determine the exact location where the problem has occurred and can respond promptly to resolve the problem.

The response of the river administrators with respect to the posted problems occurs as follows. When there is a new post by a user, the notification function notifies the river administrator about it by e-mail. The river administrator then promptly views the post. However, since the posted information may include information not suitable for public release, the entries are made public/private based on the judgment of the river administrator. After viewing the post content, the administrator responds to the problem and can notify the contributor about the action being taken through a comments function incorporated in the system. In addition, the river administrator can search for previous related posts on the posting management screen.

Figure 1 shows the home screen (new posts) of the RIMS. A list of the recent posts is shown as the first page so that the river administrator can promptly respond to the related problems.

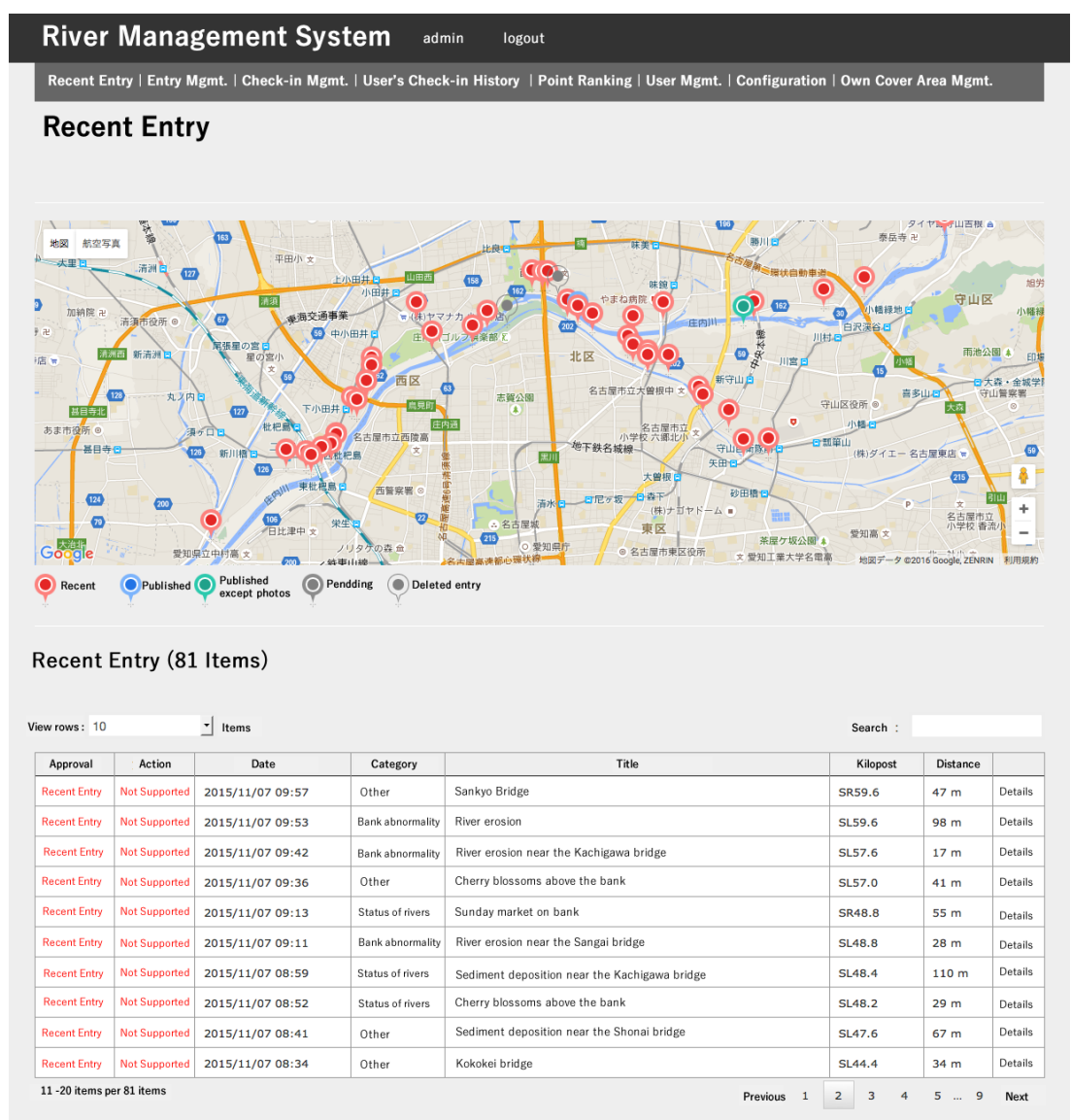

Figure 1: Top page (management of recent entries). 


\subsection{Check-in Function}

The RIMS has a check-in function based on gamification. By setting up check-in point, the administrator can confirm when someone arrives at these points, even when there are no posts related to these points. Even if there is no post in the place, there is not necessarily a problem. At this time, if there is a record checked in at that place, there is a high possibility that there is no problem. However, if there no record of a check-in, it is possible that nobody has reached the place even though an undetected problem may have occurred at that location. This makes it possible to distinguish between the places where the possibility of a problem occurring is high. One can then take measures such as ensuring intensive patrolling by river inspectors. In addition, the check-in function based on gamification gives a greater sense of satisfaction to the users.

The check-in point is set in advance by the administrator using the check-in management screen. The check-in point can be set to any arbitrary place on the map displayed on the check-in management screen. When the user activates the RIA, the 20 latest check-in points closest to the server are acquired from the server and displayed on the map. Depending on the user settings, it is possible to allow for automatic or manual check-ins. Automatic check-ins mean that the user is checked in automatically when they reach the check-in point. On the other hand, for manual check-ins, when the user reaches the check-in point, a message is displayed asking whether a check-in by the user is possible or not. Once the check-in process is complete, the data are sent to the server, and the check-in is recorded.

Figure 2 shows the check-in management screen. The banner in the map indicates the check-in point. The pink circles around the check-in points indicate the range over which check-ins are possible. The red dots indicate the check-ins posted by the user. The administrator can verify the check-in record of each user through this check-in management screen.

Figure 3 shows the screen for confirming user's behavior. During disaster patrols, the river administrator has to be able to determine the actions of the patrol staff and ensure their safety. The blue flags in the map indicate the points where users have checked in. These have been connected with a red line to denote their chronological order. The record of check-ins can confirm the movement history of each user on the user screen.

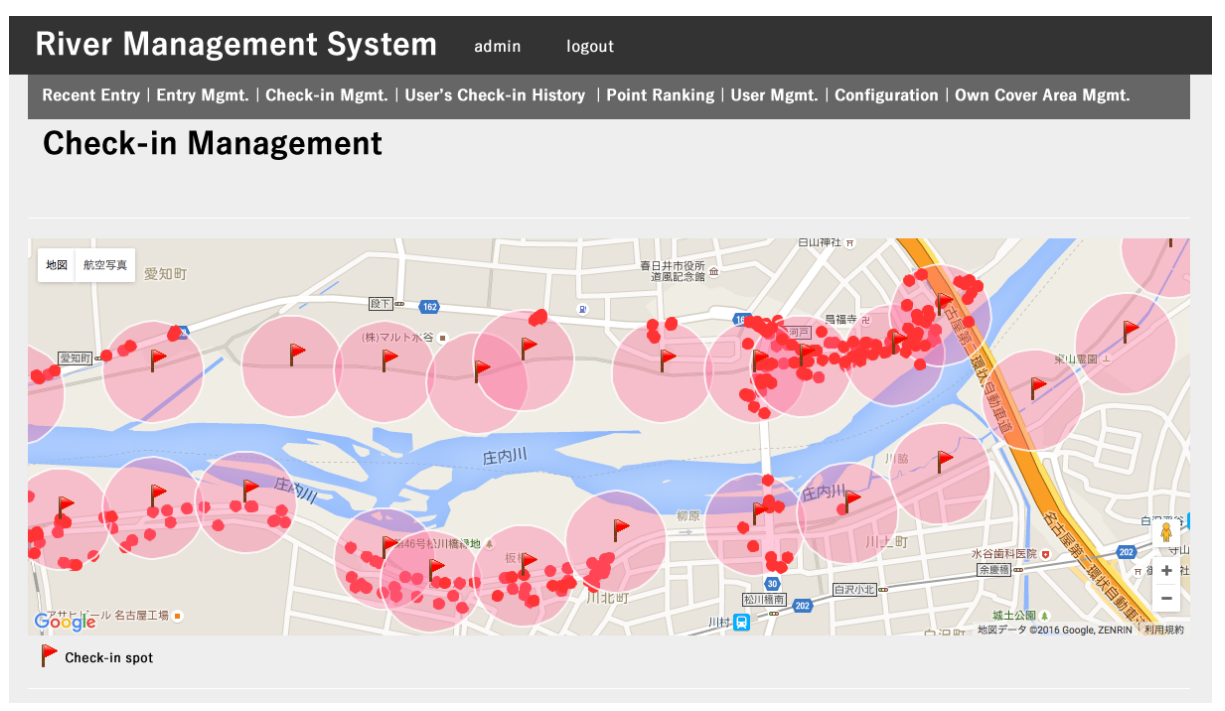

Figure 2: Check-in history management (check-in management). 


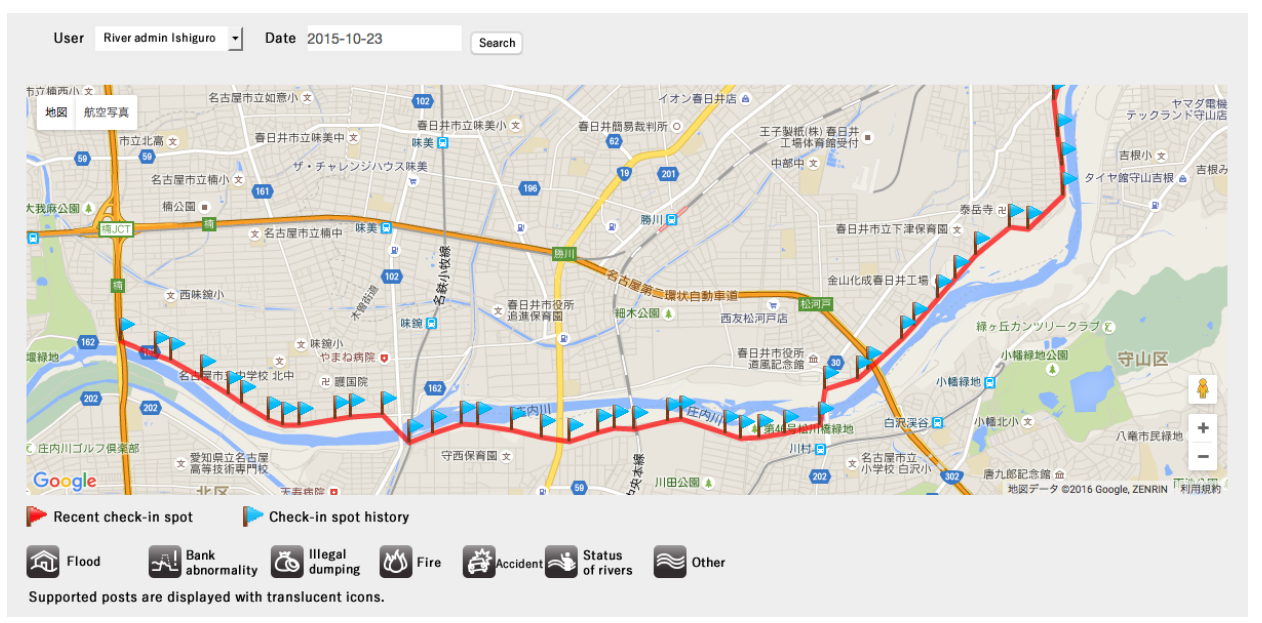

Figure 3: User check-in history.

\subsection{Point-Ranking Function}

The check-in function gives users greater mental satisfaction. However, it is necessary to provide continuous motivation to ensure that they use the service regularly. Therefore, in order to motivate the users of the RIA, we implemented the point and point ranking functions as incentive mechanisms.

The point ranking function should encourage the users to continuously use the RIA. The administrator presets the number of points to be given to a user at the time of posting and checking-in advance by the PMS. The number of points allocated at the time of checking-in can vary depending on the location. A message is displayed to the user when the posting and check-in processes are completed, and the related points have been allocated. Furthermore, using the point ranking system, the total number of points accumulated by a user is displayed in the form of a ranking, which is visible to all the users.

\section{System Configuration}

\subsection{Platform of System}

As mentioned earlier, the RIMS consists of the PMS and the RIA. The PMS is a web-based system and was developed under the following environment. The server OS used was Ubuntu 14.04 LTS $^{3}$ while the Web server used was Nginx 1.4.3 ${ }^{4}$. We used Ruby 2.0.0 p481 ${ }^{5}$ and JavaScript as the programming languages. Ruby on Rails $4.0 .3^{6}$ was used as the framework while Unicorn 4.8.3 ${ }^{7}$ was used for the dispatcher. Finally, the database used was MySQL 5.5.46 ${ }^{8}$.

\footnotetext{
3 Ubuntu, http://www.ubuntu.com/

4 nginx, http://nginx.org/

5 Ruby, https://www.ruby-lang.org

6 Ruby on Rails, http://rubyonrails.org/

7 Unicorn, http://unicorn.bogomips.org/

8 MySQL, https://www.mysql.com/
} 
The RIA is an iOS ${ }^{9}$ application and was developed under the following environment. The programming language used was Swift $2.0^{10}$, and the software was developed for iOS versions 8 and higher. The software development kit used was iOS SDK 9.1, and the library management tool used was Cocoa Pods 0.38.2.

\subsection{Configuration of Entry Management System}

The PMS allows river administrators to quickly become aware of any river-related problems based on the messages posted by local residents and respond to them appropriately. All the posted information is stored in a database, which allows accidents and incidents of illegal dumping to be determined from the posting history.

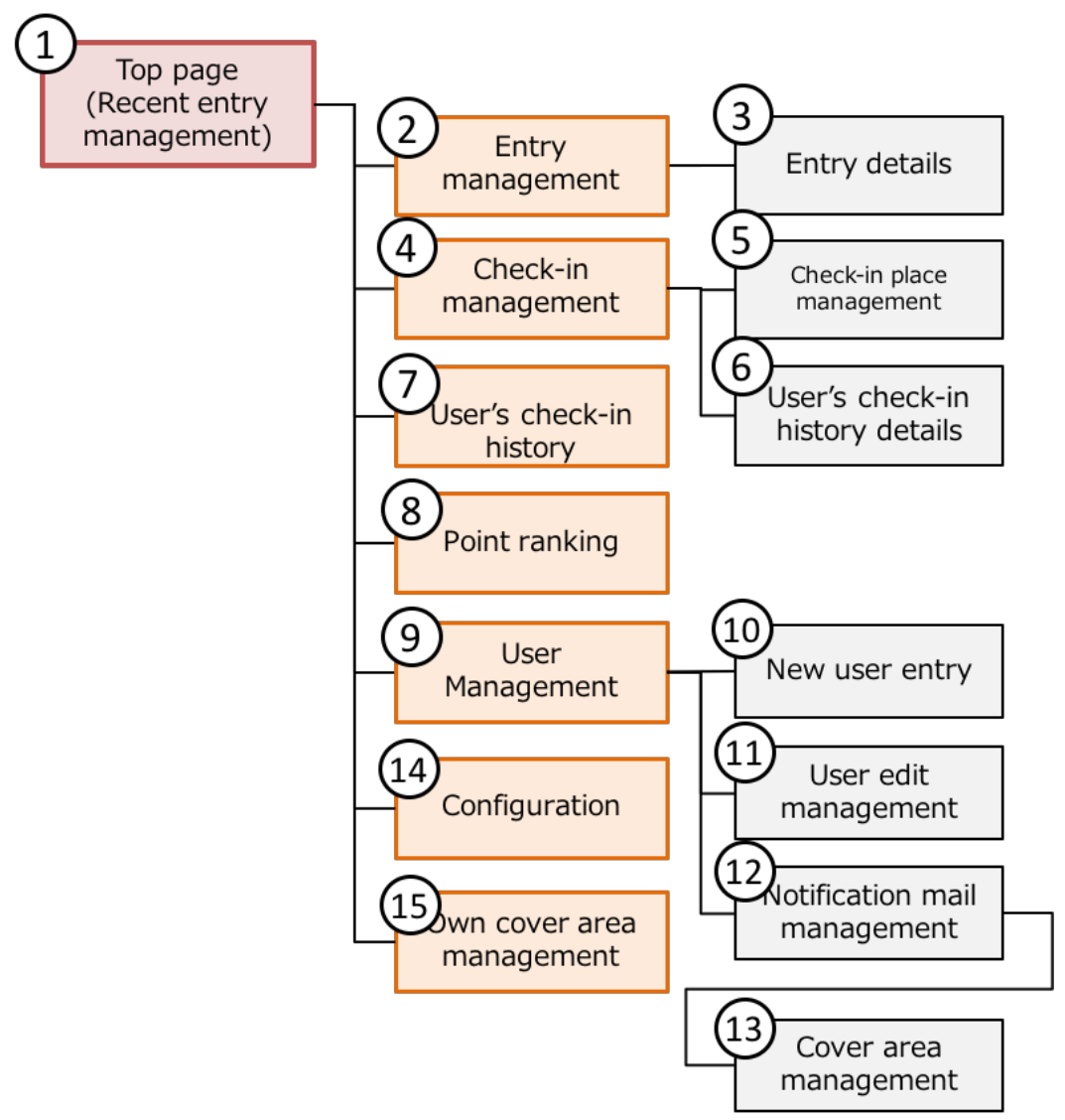

Figure 4: Page structure of River Management System.

Figure 4 shows the page structure of the PMS. The home screen (list of new posts) in Option 1 of Figure 4 is the first page to be displayed after the user logs in (Figure 1). A list of the posts that need to be processed is displayed, making it possible to respond to them promptly. A chronological list of the new postings as well as the posts requiring a response is shown. In addition, a map is displayed at the top, and the places corresponding to the new posts are shown on the map using different icons depending on the post category.

\footnotetext{
9 iOS - Apple, http://www.apple.com/jp/ios/

10 Swift, https://swift.org/
} 
The entry management page in Option 2 of Figure 4 allows for all the entries to be viewed. The user can search through all the posts as well as browse through them using the displayed map and the list view. Further, the user can switch from the detailed links view to the post details page. The user can also download the post list in CSV format.

The entry details page in Option 3 of Figure 4 displays the details of each post. Specifically, the posting status, title, posting user, contribution date/time, posting category, distance from the kilo-post, and any related comments are displayed. Whether a post or comment is to be made visible or not can be decided using the Post details screen.

Option 4 of Figure 4 shows the screen for managing (creating, editing, and deleting) the check-in spots (Option 5 of Figure 4) and browsing the checked-in users (Option 6 of Figure 4) (see Figure 2).

The user behavior check option in Option 7 of Figure 4 allows one to view the check-in history of each user (Figure 2). By specifying the date and time, one can search through the check-in history of any user. On the map, the check-in history of the user is displayed using icons, which are connected by a red line to show their chronological order. This function is for checking the present position of the patrol staff and confirming their safety when the staff is in the field responding to a natural disaster or a similar event.

The point-ranking option in Option 8 of Figure 4 shows the points accumulated by the various users and their corresponding ranks. Points are awarded to the users each time they post or check-in.

Using the user management option in Option 9 of Figure 4, all the users of the RIA can be managed. This option not only allows new users to be registered (Option 10 of Figure 4) but also allows their information to be edited (Option 11 of Figure 4). It also allows their privileges to be controlled. Only users with the required administrative authority can log in and use the PMS. In the mail notification management section (Option 12 of Figure 4) of the user management option, it is possible to set the range of responsibilities of the administrator so as to notify the specific supervisor in charge by e-mail when new arrivals are posted Yes (Option of 13 Figure. 4).

The various settings of Option 14 of Figure.4, Category setting, Comment standard size setting, Check-in radius setting, Set point to post, and Posting authorization range setting can be set. The category setting allows one to allocate categories to the posts while the comment standard size setting allows the length of the comments that can be sent to the contributors to be set. The check-in radius setting allows a verifiable range of distances from the center of the check-in point to be set. The points setting for postings allows the points to allocated to the users when they post to be set. The same number of points are allocated to each user. Finally, the posting permission range setting allows the range over which posts can be made to be set. In this system, we focused on posts related to rivers and hence restricted any posts related to areas that are not near rivers. Specifically, posts corresponding to locations in a certain distance away from the kilo-posts along the river are not allowed by the system. The kilo-post is a sign indicating the distance from the mouth of the river, starting from the mouth of the estuary. For instance, kilo-posts are installed along the Shonai River at intervals of $0.2 \mathrm{~km}$. Posts that correspond to kilo-posts outside the permitted range are not accepted. The acceptable radius is the same for all the kilo-posts.

Using the own cover area management option in Option 15 of Figure 4, the logged-in user can 
set the range for which they receive a notification mail whenever a new post is made.

\subsection{Structure of River Information Application}

The RIA is an information gathering smartphone application that allows residents living around rivers to readily post river-related information to the PMS. Figure 5 shows the page structure of the RIA.

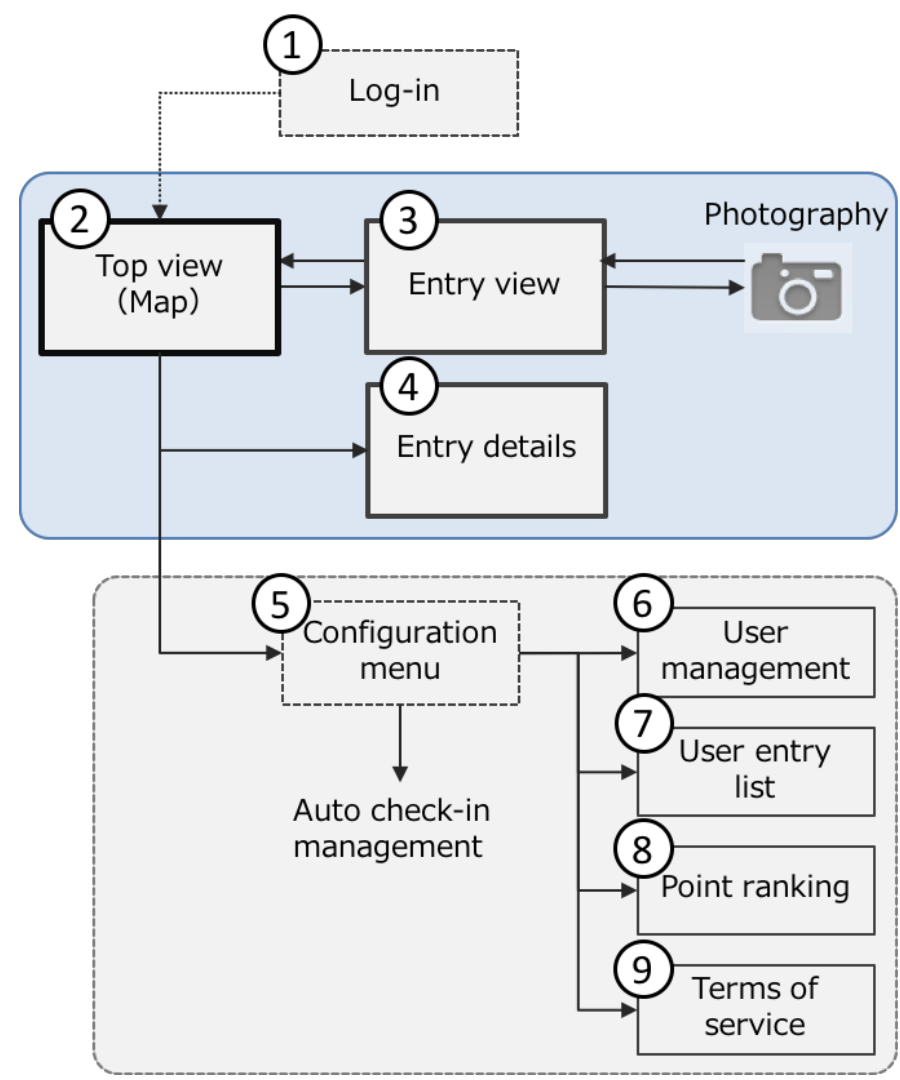

Figure 5: Page structure of RIA.

After the initial application startup, the user must register and log in (Option 1 of Figure 5).

The top screen in Option 2 of Figure 5 shows a map based on the current location of the user, as determined by the GPS function of their smartphone. Figure 6 shows the top screen of the RIA. In this screen, the nearby check-in points as well as the existing posts are indicated using icons. The flag icons shown in Figure 6 indicate check-in points while the red circles surrounding the check-in points indicate the range over which checking-in is possible. At the bottom of the top screen, icons indicating the current location as well as the Update, Menu, and Post options are displayed. The current location displays the map of the area around the current position. The Update option updates the check-in spot on the map as well as the existing post-related information. The Menu option displays the setting menus and allows the user information to changed. The Post option takes the user to the submission screen.

In the Posting screen in Option 3 of Figure 5, fields for adding photographs and entering information related to the post category as well as for adding the title and related text are shown. A map indicating the posting place is also displayed. The user enters this information to make a 
new post. Photographs taken from the Posting screen as well as those obtained using the smartphone camera can be used.

Using the Posting details option in Option 4 of Figure 5, it is possible to view the already published posts and their contents.

Using the Setting menu in Option 5 of Figure 5, the user is taken to the "User Information Browsing," "User Posting List," "Point Ranking," and "Automatic Check-in Settings" options. The Usage Agreement can also be viewed here. The User Information Browsing option in Option 6 of Figure 5 allows the users to view and edit their personal information. The User Submission List option in Option 7 of Figure 5 shows the list of posts made by the user. The individual posts can be accessed from the Post Details screen. The Point Ranking option in Option 8 of Figure 5 shows the rankings of all the users based on the points accumulated by them after posting or checking-in. The Automatic Check-In Setting option allows the check-in option to be set to automatic or manual. Automatic check-ins mean that the user is checked in automatically when they reach the check-in point while under the manual option, when the user reaches the check-in point, a check-in confirmation screen is displayed, and checking-in must be performed manually. The Terms of Service option in Option 9 of Figure 5 shows the terms of use of the RIA.

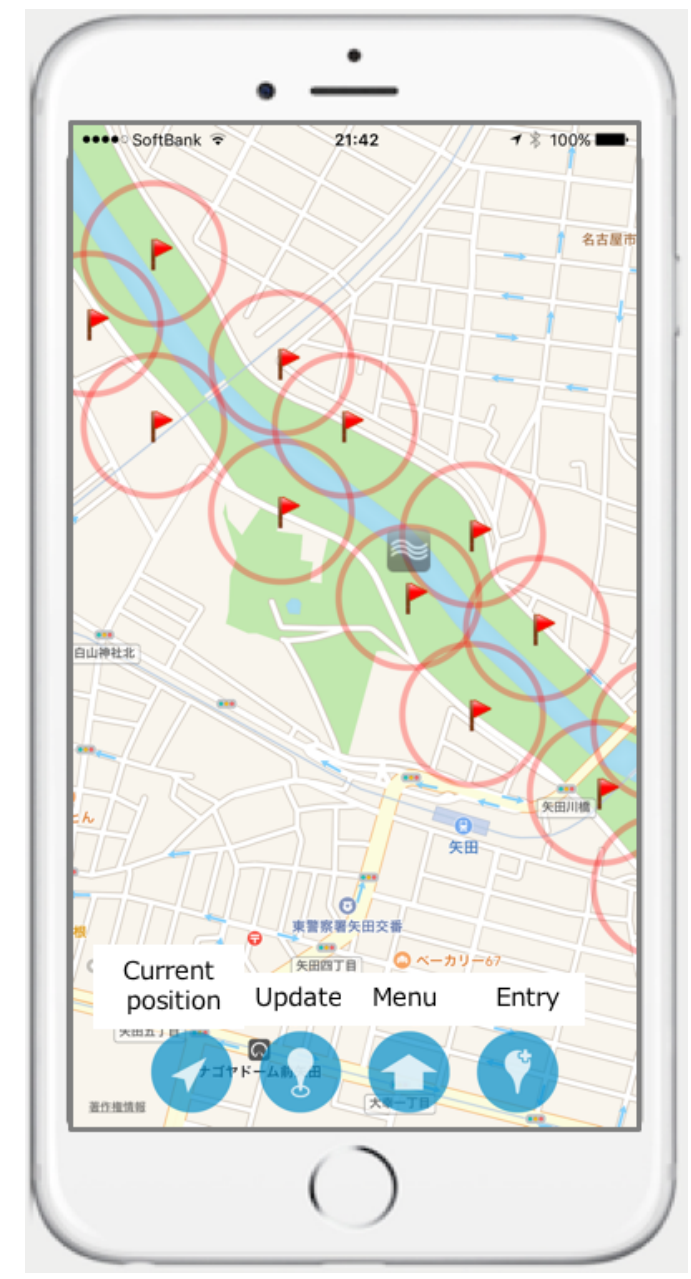

Figure 6: Main screen of RIA. 


\section{Field Tests}

\subsection{Overview of Field Tests}

In order to evaluate the RIMS, field tests were performed with the cooperation of the river administrator and patrol staff of the Shonai River Office (Figure 7). Confirm the operation of the function based on the three problems for improving problems around the river.

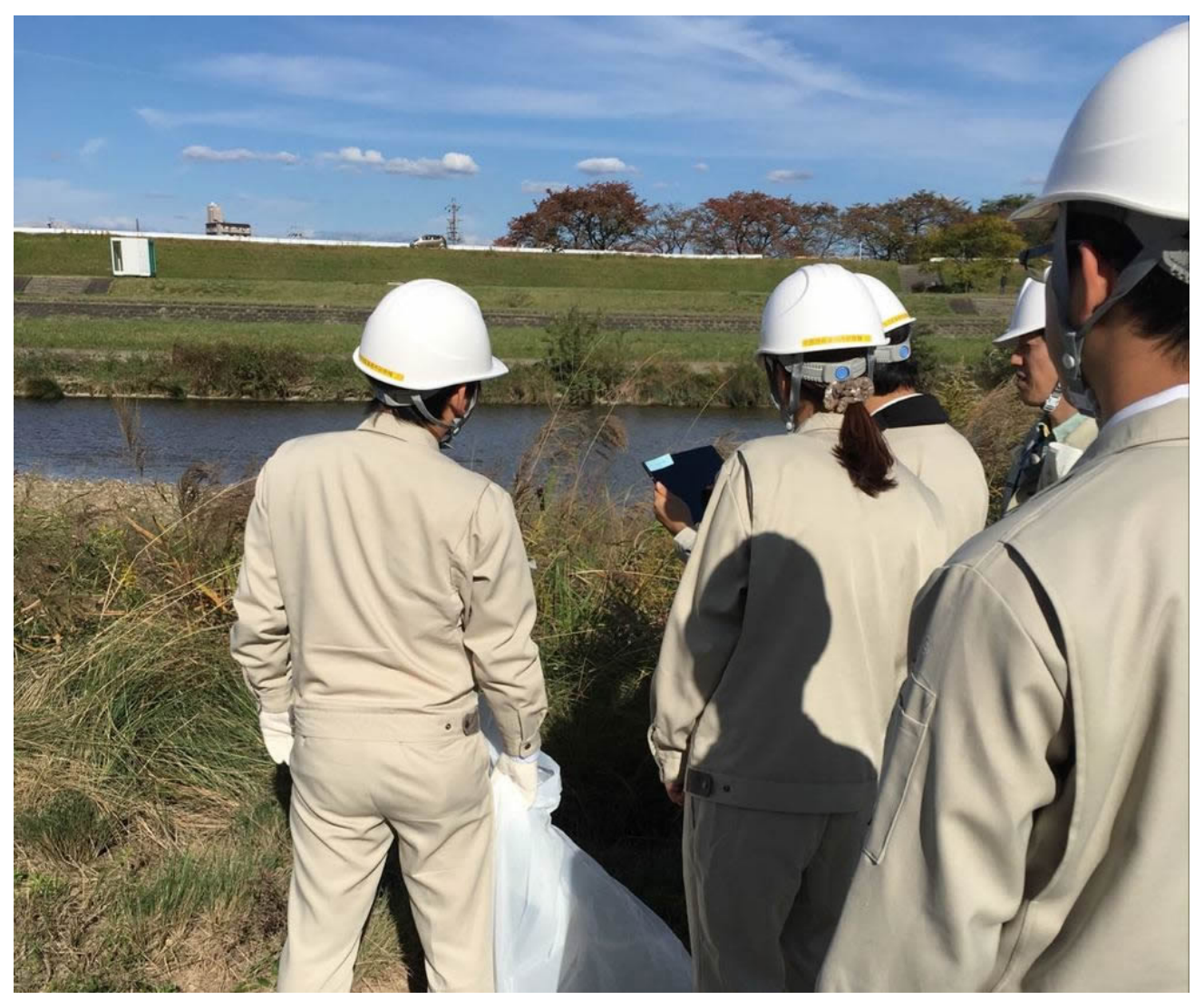

Figure 7: Field tests performed by river administrator and patrol staff.

The RIA was used by the patrol staff while the river administrator tested the posting and check-in function as well as the point ranking function of the PMS. The tests were performed from October 14, 2015 to November 7, 2015 in the vicinity of the Shonai River and the Yada River (a tributary of the Shonai River), which are under the jurisdiction of the Shonai River Office. The equipment used included an iPhone 6 Plus smartphone, an iPad Air 2 notebook, and iPad Mini 4 and iPad 2 tablets. A Wi-Fi router was used for connecting to the internet.

The patrolling of the river is usually done by the replacement of two people. The number of times patrolling was performed using the RIA during the field tests varied depending on the patrol staff members. Also, when submitting problems of rivers, one of the two patrol stations posts, and the number of postings are biased. Therefore, the numbers of submissions and check-ins varied greatly depending on the patrol staff members. 
Table 1: Results of field tests of RIMS (October 14, 2015 to November 7, 2015)

\begin{tabular}{l|rr|r}
\hline Tester & Number of check-ins & Entries & Points \\
\hline Patrol member A & 59 & 20 & 1,590 \\
Patrol member B & 12 & 3 & 270 \\
Patrol member C & 119 & 17 & 2,040 \\
Patrol member D & 75 & 3 & 900 \\
Patrol member E & 293 & 0 & 2,930 \\
\hline (Total) & 558 & 43 & - \\
\hline
\end{tabular}

\subsection{Test of Entry Function}

We first tested the posting function of the RIMS in terms of whether it allows for the receiving of information in an accurate manner from the residents living around the river. This was done with help of the patrol staff during patrolling, who used the RIA to report the conditions around the river.

Table 1 shows the results of the posting test. Regarding the patrol staff $B$ and the patrol staff $D$ in Table 1, one pair of two patrol stations has posted, and the number of submissions has decreased. A total of 43 entries were made in PMS operated by the river administrator. For each post, we confirmed that the correct information regarding the title, posting user, contribution date/time, posting category, and the nearest kilo-post had been recorded appropriately. We also sent a comment from the administrator and confirmed that the comment was correctly displayed on the device used by the poster. Further, using the administrator settings, we confirmed that we could decide whether a particular post was to be displayed to the rest of the users or not. We found that there were cases where the post category was reflected incorrectly. This problem was subsequently correct, as explained in Section 5.5. Thus, it was confirmed that the posting function, which is the basic function of the RIMS, works as desired, with the only issue being that, in a few cases, the correct post category was not displayed.

\subsection{Test of Check-in Function}

With a patrol staff member, carry an RIA at the time of patrol work and confirm that check-in is done when it reaches the check-in point set as a patrol route.

The test to evaluate the check-in function was performed as follows. In order to evenly distribute the check-in points along the river basin, we set 694 locations at the same points as the kilo-posts. Further, the check-in radius was set to $100 \mathrm{~m}$.

In Table 1, since the patrol staff member B made fewer patrols using the RIA, their number of check-ins was also lower. In addition, the patrol staff member E only performed the check-in function test and did not perform the contribution function test described above. Hence, a total of 558 check-ins were recorded.

We confirmed the check-in record of the patrol staff in the RIA using the PMS, which is to be operated by the river administrator and User action confirmation. We verified the check-in data against the patrol staff's patrol route for the corresponding day. Because the check-in function uses the GPS function of smartphones, it is affected by factors such as the reception quality of the GPS signal and the accuracy of the GPS function of the smartphones. 
There were cases where it was not possible to check in due to the accuracy of the GPS of the smartphone, but when recognizing that the smartphone entered the check-in setting area, I confirmed that I can check in normally. The correspondence and the like about the cases that cannot be checked in will be described in Section 5.5. Thus, we were able to confirm that the check-in function of the RIMS works as expected.

\subsection{Field Tests of Point and Point Ranking Functions}

Next, the point and point ranking functions were tested. Here, the officers out on patrols were asked to use the RIA to make posts and perform check-ins and subsequently to verify their point and point rankings. The number of points awarded was set at 50 for each post and 10 for each check-in.

In Table 1, the total number of check-ins was 558 while the total number of postings was 43 . The postings and check-ins made by the patrol staffs using the RIA and their point rankings were confirmed using the PMS operated by the river administrator. We also confirmed that the point rankings were displayed and updated on every terminal on which the RIA was installed. Thus, we could confirm that the points and point ranking functions of the RIMS function as desired.

\subsection{Test of Gamification}

Figure 8 shows the time series change of points of users. Patrol members A, C, and D have gradually gained points. On the other hand, patrol members B and $\mathrm{E}$ have little change. Patrol member E got points suddenly because he moved a long distance by car for patrol.

In the RIA, the point ranking function described in Section 3.3 allows a user to check the number of points for another users. Patrol member $\mathrm{C}$ can check the number of points of patrol member $\mathrm{A}$ with the point ranking function. The number of points of the patrol member $\mathrm{C}$ gradually rises, it can be considered as an action conscious of the point number of the patrol A and it can be seen as the effect of the gamification. Patrol members B and E can be seen as not having much effect of giving points.

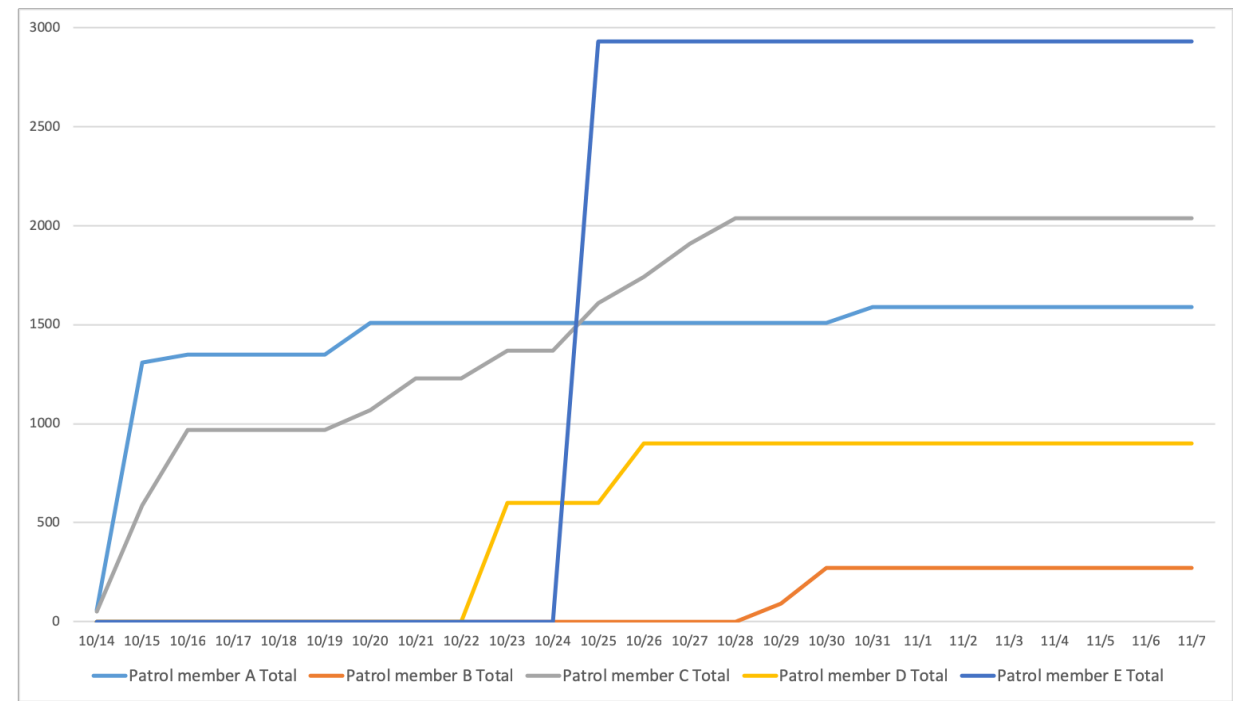

Figure 8: The time series change of points (October 14, 2015 to November 7, 2015) 


\subsection{Discussion}

Based on the test results described above, it was confirmed that the posting, check-in, point, and point ranking functions work as expected. In addition, with the help of the patrol staff, who performed the field tests, and the river administrator, the post approval and e-mail notification functions, implemented as per the manager's request, were also evaluated. In addition, the effectiveness of the check-in, point, and point ranking functions in motivating the users to participate was also evaluated. Thus, it was confirmed that the functions implemented in this study are effective.

In addition, we received the following feedback from the patrol staffs.

1. On the posting screen, the size of the map of the "posting place" is small, and its length needs to be increased.

2. The posting screen should allow for multiple photographs to be shared.

3. On iPads, the post "Category" could not be changed when making posts (error related to posting function).

4. With respect to the check-in function, the GPS sensitivity was poor and there were instances where it was not possible to check-in (error related to check-in function).

Based on this feedback, we plan to make the following changes to the developed system:

(1) Although it is feedback by valuable feedback on the site and valuable opinion, it is necessary to make modifications in consideration of usability of the entire user interface. It is not a problem of fundamental function. It is not an urgent problem. For future work, we will make comprehensive modifications along with other requests related to the user interface.

(2) It was observed that there were cases where the ability to submit multiple photographs was necessary owing to issues occurring at the corresponding site. Adding this functionality will require modifications such as changes in the database structure. However, since this problem does not affect the basic functions of the system, we will respond to it in the future.

(3) It was found that, after category selection, while scrolling through the screen, the selected category is initialized. This problem was specific to iPads. The problem was corrected by referring to the specifications for the iPad models used and tests were performed to confirm that the issue has been resolved.

Regarding the poor GPS sensitivity (reception) of device 4, in addition to the accuracy of the GPS signal of the device, the state of the device itself (how it is held and whether it is being moved or not) as well as the status of the internet connection (speed and reception quality) and factors such as the weather conditions would affect the GPS signal. Therefore, even if the GPS sensitivity is poor, it can be related to several factors. Further, since this issue did not affect the basic functions of the system, we will deal with it in a future study. This will be done by testing various devices under a range of conditions related to the GPS signal.

To conclude, it was confirmed that the basic functions incorporated in the proposed system 
worked as expected. Further, an e-mail notification function, which is not included in the existing participatory sensing services, was added based on a request by river administrators. It can be expected that correspondence becomes possible. In addition, it can be expected that the users of the system will contribute more readily and for longer periods owing to the gamification-based incentive mechanisms incorporated in the system.

\section{Conclusions}

The illegal dumping of garbage, flooding, and levee and bridge failures are some of the common problems related to rivers. To be able to tackle these issues effectively, it is necessary to understand and respond to them with speed. In addition, future measures are required. However, since river basins can be very large, it is difficult for the river management staff to notice and respond to all river-related issues promptly. In this study, we developed an RIMS composed of PMS and RIA for the Shonai River. Through this development, the river information posting system basis for the local residents such as cooperating organizations around the river to post circumstances around the river by smartphones, promptly confirm the posting by the local residents and promptly respond. We performed field tests in the Shonai River basis and were able to confirm that basic functions of the developed system work as expected.

We will present future issues. Adjust usability based on user's feedback to make it more user-friendly system. Based on the development of this system, it is necessary to construct a system that can operate in multiple rivers.

\section{Acknowledgements}

This work was supported by a Grant-in-Aid for Scientific Research (KAKENHI) (grant number JP18K45678) from the Japan Society for the Promotion of Science and a CREST grant (grant number JPMJCR15E1) from the Japan Science and Technology Agency.

\section{References}

[1] J. Burke, D. Estrin, M. Hansen, A. Parker, N. Ramanathan, S. Reddy and M. B. Srivastava: Participatory Sensing, World Sensor Web Workshop, ACM Sen Sys 2006, Colorado, Oct 2006.

[2] N. D. Lane et al.: A survey of mobile phone sensing, IEEE Comm. Mag., vol. 48, no. 9, pp. 140-150, Sep. 2010.

[3] S. S. Kanhere: Participatory sensing: Crowdsourcing data from mobile smartphones in urban spaces, in Proc. IEEE MDM, pp. 3-6, 2011.

[4] H. Lu, D. Frauendorfer, M. Rabbi, M. S. Mast, G.T. Chittaranjan, A. T. Campbell, and T. Choudhury: Stress Sense: Detecting Stress in Unconstrained Acoustic Environments using Smartphones, ACM International Conference on Ubiquitous Computing (Ubi Comp 2012), pp.351-360, 2012.

[5] R. Li Kam Wa, Y. Liu, N. D. Lane, and L. Zhong: Mood-Scope: Building a Mood Sensor 
From Smartphone Usage Patterns, The 11th ACM International Conference on Mobile Systems, Applications, and Services (Mobi Sys 2013), pp.389-402, 2013.

[6] M. Azizyan, I. Constandache, and R. R. Choudhury: Surround Sense: Mobile Phone Localization via Ambience Fingerprinting, The 15th Annual International Conference on Mobile Computing and Networking (Mobi Com 2009), pp. 261-272, 2009.

[7] Kazemi, Leyla, and C. Shahabi: Geocrowd: enabling query answering with spatial crowdsourcing, Proceedings of the 20th International Conference on Advances in Geographic Information Systems. ACM, 2012.

[8] S. Deterding, D. Dixon, R. Khaled, and L. Nacke: From game design elements to game fulness: defining "gamification", In Proc. of the 15th Int'l Conf. on Academic MiTrek: Envisioning Future Media Environments, pp. 9-15, 2011.

[9] S. Deterding, M. Sicart, L. Nacke, K. O'Hara, and D. Dixon: Gamification: using game-design elements in non-gaming contexts, In Proc. of the 2011 Int'l Conf. on extended abstracts on Human factors in computing systems (CHI), pp. 2425-2428, 2011.

[10] Knight, James F., et al.: Serious gaming technology in major incident triage training: a pragmatic controlled trial., Resuscitation 81.9, pp.1175-1179, 2010.

[11] L. V. Ahn and L. Dabbish: Labeling images with a computer game, In Proc. of the ACM SIGCHI Conf. on Human factors in computing systems, pp. 319-326, 2004.

[12] J. S. Lee and B. Hoh: Dynamic pricing incentive for participatory sensing. Pervasive and Mobile Computing, Vol. 6, No. 6, pp. 693-708, 2010.

[13] D. Yang, G. Xue, X. Fang, and J. Tang: Crowdsourcing to smartphones: incentive mechanism design for mobile phone sensing, in Proc. ACM Mobi Com, 2012, pp. 173-184, 2012.

[14] I. Krontiris and A. Albers: Monetary incentives in participatory sensing using multi-attributive auctions, International Journal of Parallel, Emergent and Distributed Systems, Vol. 27, No. 4, pp. 317-336, 2012.

[15] L. G. Jaimes, Idalides: A location-based incentive mechanism for participatory sensing systems with budget constraints, In Proc. of the IEEE Per Com, pp. 103-108, 2012.

[16] I. Schweizer, C. Meurisch, J. Gedeon, R. Bartl, and M. Muhlhauser, Noisemap: Multitier incentive mechanisms for participative urban sensing, in Proc. ACM Phone Sense, pp. 9:1-9:5, 2012.

[17] King. S, and Brown. P.: Fix my street or else: using the internet to voice local public service concerns, The International Conference on Theory and Practice of Electronic Governance, pp.72-80, Macau, 2007. 